\title{
Use of a novel through-the-needle biopsy forceps in endoscopic ultrasound
}

Authors

Institutions
Mohammad H. Shakhatreh' ${ }^{1}$ Sohrab Rahimi Naini ${ }^{1}$, Alan A. Brijbassie ${ }^{1}$, Douglas J. Grider ${ }^{2}$, Perry Shen ${ }^{3}$, Paul Yeaton ${ }^{1}$

Institutions are listed at the end of article. submitted

30. November 2015

accepted after revision

22. January 2016

\section{Bibliography}

DOI http://dx.doi.org/

10.1055/s-0042-101941

Published online: 30.3.2016

Endoscopy International Open

2016; 04: E439-E442

(c) Georg Thieme Verlag KG

Stuttgart · New York

E-ISSN 2196-9736

\section{Corresponding author}

\section{Mohammad H. Shakhatreh,}

\section{MD, MPH}

3 Riverside Circle

Roanoke, VA 24014

USA

Fax: +1-540-985-9419

mhshakhatreh@carilionclinic. org
Background and aims: Pancreatic cysts are becoming more common. Their differential diagnosis includes benign, premalignant, and malignant lesions. Distinguishing the type of cyst helps in the management decision making. We report on a novel tissue acquisition device for pancreatic cysts.

Methods: Data on two patients who underwent endoscopic ultrasound (EUS) - guided fine-needle aspiration with a new micro forceps device are presented.

Results: Two patients had large pancreatic cystic lesions in the pancreatic head. Linear EUS was

\section{Introduction}

$\nabla$

Pancreatic cysts are increasingly being identified in asymptomatic patients. Overall, the sensitivity of endoscopic ultrasound with fine-needle aspiration (EUS-FNA) is modest, but the specificity for malignancy is high [1]. We report our results with the use of a new device, the Moray micro forceps (US Endoscopy, Mentor, Ohio, USA), to perform EUS-FNA in two patients having pancreatic cysts incidentally noted on cross-sectional imaging. The purpose of the study was to establish the technical feasibility of using this new tool to acquire pancreatic cyst wall tissue and aid in diagnosis and management.

\section{Case 1}

\section{$\nabla$}

An 81-year-old woman with chronic obstructive pulmonary disease (COPD) underwent cross-sectional imaging of the chest because of shortness of breath. A 7.0 $\times 3.7-\mathrm{cm}$ multiloculated cystic collection of the pancreatic head was incidentally defined, with a normal-appearing body and tail ( $\bullet$ Fig. 1). The patient was referred for EUS with possible FNA, which demonstrated a $4.4-\mathrm{cm}$ anechoic septate cystic lesion containing debris. performed, and tissue samples were obtained with the Moray micro forceps through a 19-gauge needle. In both patients, mucinous columnar epithelium lined the cystic walls. One patient underwent surgical resection, and the other elected surveillance. Examination of the surgical specimen from the first patient confirmed the cyst was a side-branch intraductal papillary mucinous neoplasm (IPMN), gastric type.

Conclusions: The Moray micro forceps is a new tool that can be used to help determine the nature of pancreatic cysts and aid in their risk stratification and management.

There was no invasion of the adjacent vasculature, and no intramural nodularity was observed. A 19-gauge needle (Cook Medical, Bloomington, Indiana, USA) was advanced into the fluid collection; however, the fluid was too viscous to aspirate. A Moray micro forceps ( $\bullet$ Fig. 2 ) was repetitively advanced through the 19-gauge needle to obtain multiple specimens ( Video 1 ).

The microbiopsy specimens yielded fragments of mucinous columnar epithelium and mucoid material ( Fig.3). There was mild focal pseudo-stratification of the nuclei because of tangential sectioning, so the basal orientation of the mucinous epithelial nuclei in the strips was maintained. No evidence of cytologic atypia, pleomorphism, or increased mitotic activity was found. Based on these findings, the pathologic diagnosis was mucinous cystic tumor, probably intraductal papillary mucinous neoplasm (IPMN) given the location in the head of pancreas and the radiologic findings.

The patient was referred for pancreaticoduodenectomy, which confirmed a side-branch IPMN, gastric type, with intermediate to focal high grade dysplasia. Finding a higher grade lesion after initial cytology or biopsy is common, as in this case (๑ Fig.4, $\bullet$ Fig.5). 


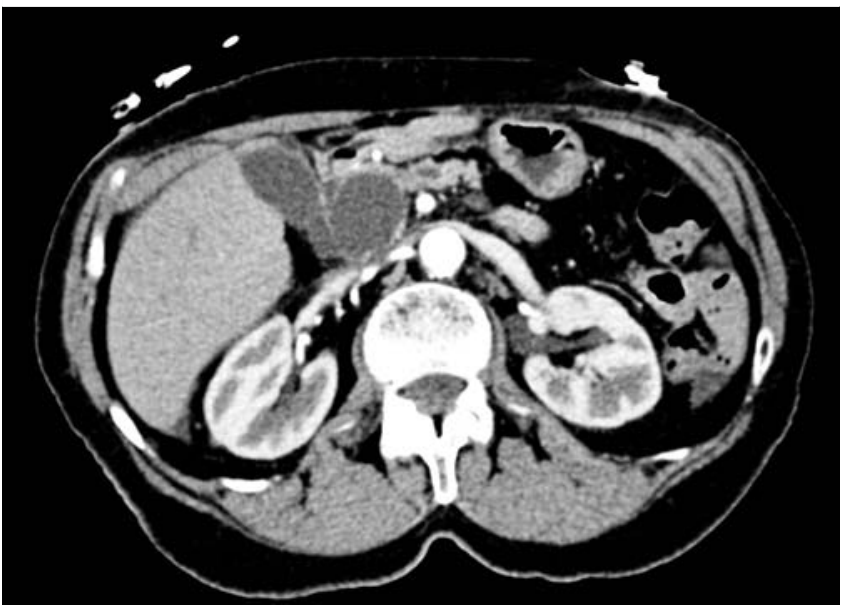

Fig. 1 Axial computed tomographic scan demonstrating a 3.7-cm cystic lesion in the head of the pancreas.

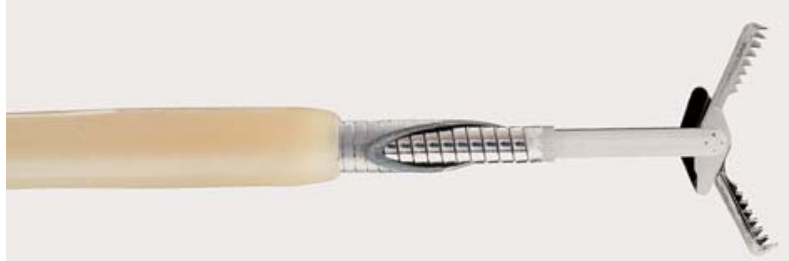

Fig. 2 The Moray micro forceps.

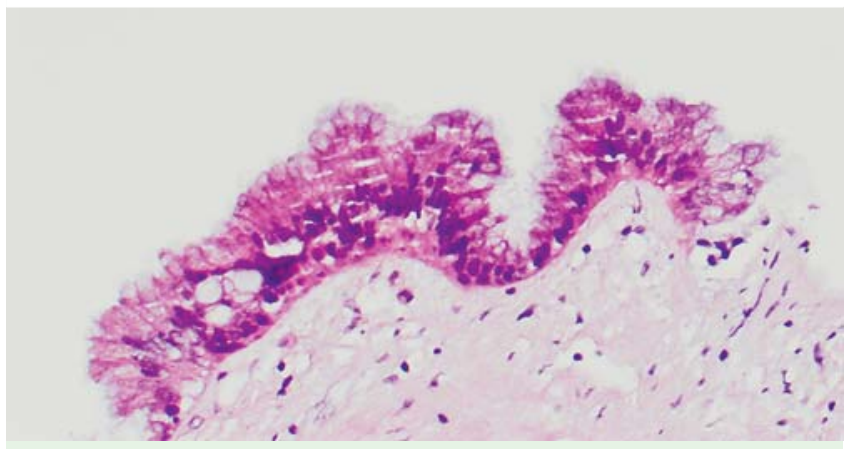

Fig. 3 Microbiopsy specimen, case 1 ( $\times 20)$. Mucinous gastric foveolar neck-type epithelium with minimal nuclear enlargement and normal nuclear-to-cytoplasmic ratio, favoring intraductal papillary mucinous neoplasm, gastric type, negative for dysplasia.

\section{Case 2}

$\nabla$

A 78-year-old man with morbid obesity was admitted to the hospital for work-up of abdominal pain. Cross-sectional imaging demonstrated numerous pancreatic cystic lesions, the largest of which was a $5.0 \times 4.7 \times 4.4-\mathrm{cm}$ septate cyst involving the uncinate process and head of the pancreas ( $\bullet$ Fig. 6 ).

EUS confirmed the presence of numerous pancreatic cystic lesions. The main pancreatic duct was normal in diameter. A 19gauge needle (Cook Medical) was advanced into the largest cystic lesion, and fluid was aspirated. A Moray micro forceps (US Endos-

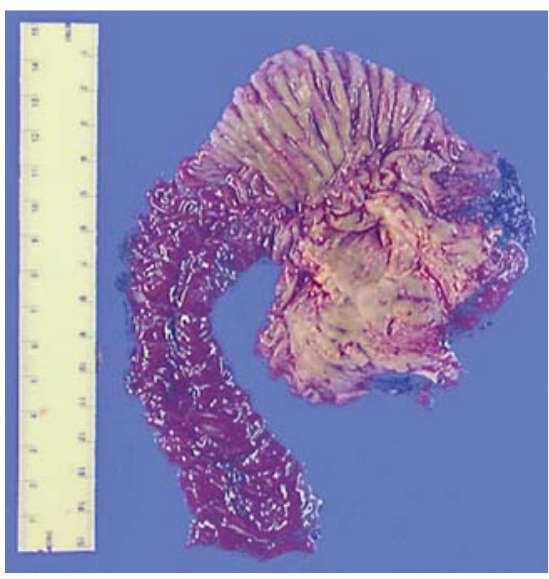

Fig. 4 Gross pathology specimen, case 1 .

copy) was repetitively advanced through the 19-gauge needle to obtain multiple specimens.

The microbiopsy specimens showed fragments of mucinous columnar epithelium and rare fragments of mucus, consistent with the clinical impression of a side-branch IPMN ( $\bullet$ Fig.7). The patient declined surgical intervention after discussion with the pancreatic surgeon because of his multiple medical co-morbidities.

\section{Discussion \\ $\nabla$}

Pancreatic cysts are incidentally found in about $2.5 \%$ of patients undergoing cross-sectional imaging for reasons not related to the pancreas [2,3], and this finding is strongly correlated with advancing age. The prevalence increases in patients identified as being at high risk for pancreatic tumors based on their genetic and familial background [4]. In an autopsy study, 186 cystic lesions were found in 73 of 300 consecutive examinations (prevalence of $24.3 \%$ ) [5]. An advantage of EUS over cross-sectional imaging is that tissue samples can be obtained when needed. Not all cysts require tissue sampling; the latest American Gastroenterological Association guidelines suggest that a sample be obtained

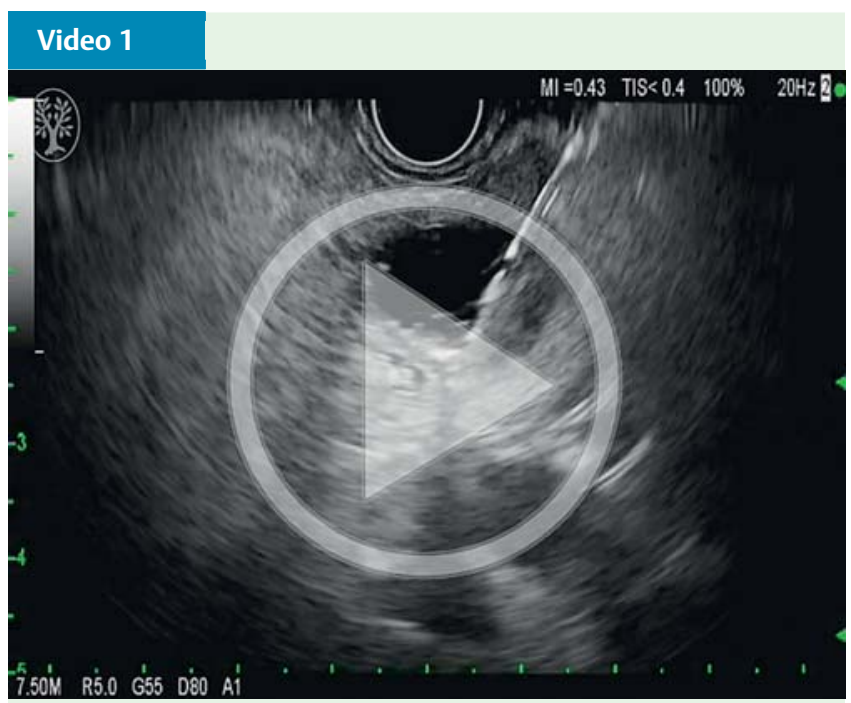

Endoscopic ultrasound with a 19-gauge needle and the Moray micro forceps. Online content including video sequences viewable at: http://dx.doi.org/ $10.1055 / \mathrm{s}-0042-101941$ 


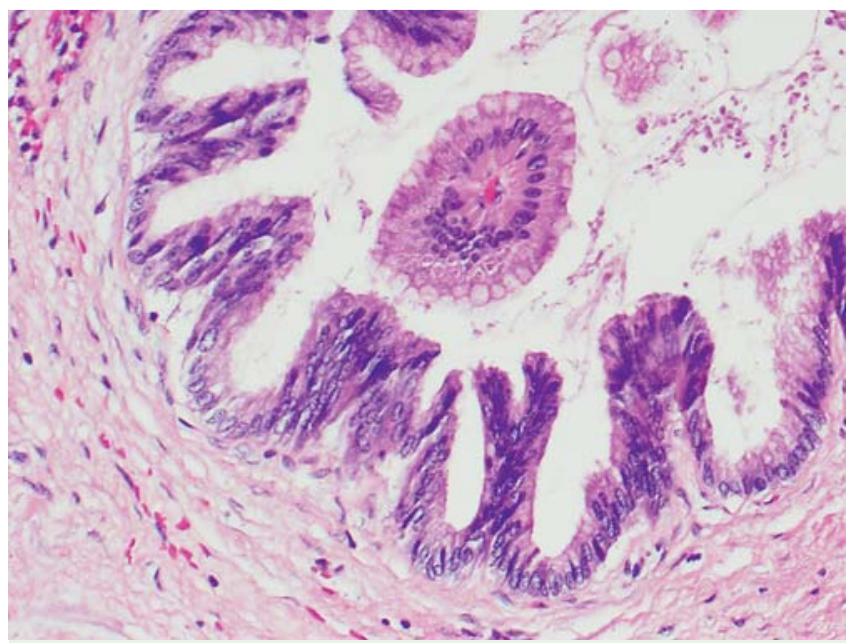

Fig. 5 Surgical specimen, case $1(\times 20)$. Gastric-type intraductal papillary mucinous neoplasm with intermediate grade dysplasia (cellular crowding, nuclear stratification, loss of polarity). Note the fibrovascular papillary core surrounded by well-oriented mucinous epithelium without dysplasia.

when a cyst enlarges to more than $3 \mathrm{~cm}$, when there is a solid component, or when the pancreatic duct is enlarged [1]. In a retrospective study of 35 surgically resected cysts larger than $3 \mathrm{~cm}$, EUS-FNA had the highest specificity (88.9\%) and magnetic resonance imaging had the highest sensitivity (100\%) [6] for the detection of high grade epithelial atypia. Despite the high specificity of EUS-FNA in diagnosing mucinous cysts and high grade atypia, its sensitivity suffers because relatively acellular samples are obtained from pancreatic cysts.

Multiple techniques have been recommended for obtaining tissue samples from pancreatic cysts. Most authorities recommend aspirating fluid from the cyst for carcinoembryonic antigen (CEA) and amylase measurement and cytologic analysis. To increase the yield of EUS-FNA and improve its sensitivity, other technical methods to obtain cyst wall tissue have been studied. In cyst wall puncture, the cyst fluid is completely aspirated, the far wall of the cyst is punctured, and the needle is moved back and forth to obtain tissue. This method has been tested with standard FNA needles [7], showing a $29 \%$ improvement in yield $(P=0.001)$ compared with CEA and cytology, and with FNA needles that have side fenestration $[8,9]$, showing a $65 \%$ sample adequacy for histopathologic diagnosis. The success rate for obtaining cyst fluid varied from $53.3 \%$ to $81 \%$.

Another tool designed to improve the yield of EUS-FNA in pancreatic cystic lesions is the cytology brush (EchoBrush; Cook Medical). Studies of this brush show contradictory results. In one study that evaluated the cytology brush in 30 patients with pancreatic cystic lesions, the brushings improved the diagnostic yield of the specimens ( 73 vs. $36 \%, P=0.08$ ); however, the technical failure rate was $27 \%$, with $13.6 \%$ morbidity and $4.5 \%$ mortality [10]. Another study examining this tool showed a significant increase in sample adequacy ( $85.1 \%$ vs. $66.3 \%, P<0.05$ ); however, intracystic bleeding occurred in three patients, and an abscess developed in one [11].

A pilot study examined the use of a SpyGlass fiberoptic probe (Boston Scientific, Natick, Massachusetts, USA) and a $220-\mathrm{cm} \times$ 0.8-mm endoscopic retrograde cholangiopancreatography (ERCP) biopsy forceps (designed for PolyScope; Lumenis Surgical, Santa Clara, California, USA) through a 19-gauge EUS needle in two patients with pancreatic cystic lesions [12]. In both patients,

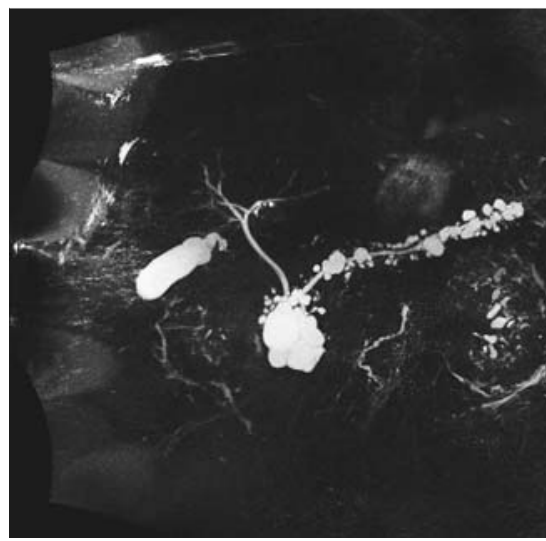

Fig. 6 Multiple pancreatic cystic lesions, case 2.

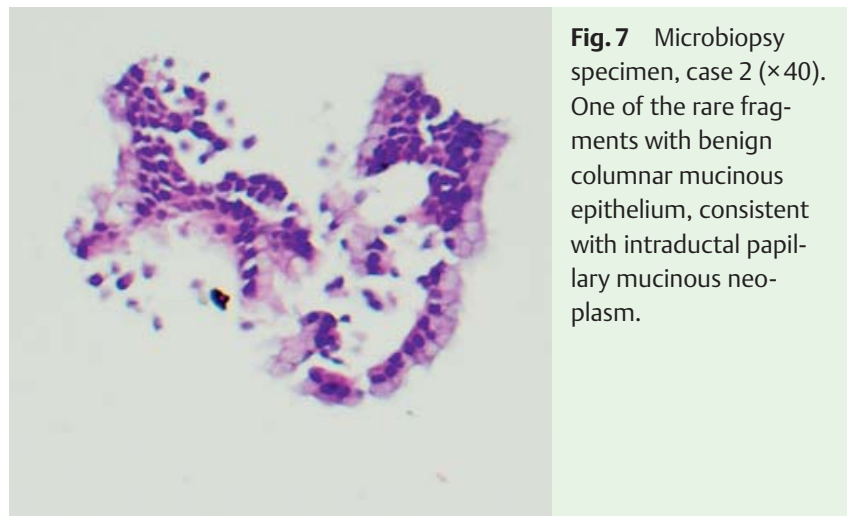

the biopsy sample findings were consistent with mucinous cystic neoplasm. Severe pancreatitis developed in one of the patients 1 month later, but it was presumed that this was unlikely to be related to the biopsy.

\section{Conclusion \\ $\nabla$}

We report use of the disposable Moray micro forceps, which is intended to sample tissue from lesions occurring within and outside the gastrointestinal tract (e.g., in the pancreas). The device length is $230 \mathrm{~cm}$, with a jaw opening width of $4.3 \mathrm{~mm}(\bullet \mathrm{Fig} .2$ ). The jaws are serrated and attached to a flexible 0.8 -mm stainless steel spring sheath that easily passes through a 19-gauge FNA needle.

\section{Competing interests: None}

\section{Institutions}

${ }^{1}$ Department of Medicine, Section of Gastroenterology and Hepatology, Virginia Tech Carilion School of Medicine and

Research Institute, Roanoke, Virginia, USA

2 Department of Pathology, Carilion Clinic and Dominion Pathology Associates, Roanoke, Virginia, USA

${ }^{3}$ Department of General Surgery, Section of Surgical Oncology, Wake Forest University School of Medicine, Winston-Salem, North Carolina, USA 


\section{References}

1 Vege SS, Ziring B, Jain $R$ et al. American gastroenterological association institute guideline on the diagnosis and management of asymptomatic neoplastic pancreatic cysts. Gastroenterology 2015; 148: 819-822

2 Laffan TA, Horton KM, Klein AP et al. Prevalence of unsuspected pancreatic cysts on MDCT. AJR Am J Roentgenol 2008; 191: 802 - 807

3 de Jong $K$, Nio CY, Hermans JJ et al. High prevalence of pancreatic cysts detected by screening magnetic resonance imaging examinations. Clin Gastroenterol Hepatol 2010; 8: 806-811

4 Canto MI, Hruban RH, Fishman EK et al. Frequent detection of pancreatic lesions in asymptomatic high-risk individuals. Gastroenterology 2012; 142: 796-804

5 Kimura W, Nagai H, Kuroda A et al. Analysis of small cystic lesions of the pancreas. Int J Pancreatol 1995; 18: $197-206$

6 Chebib I, Yaeger K, Mino-Kenudson M et al. The role of cytopathology and cyst fluid analysis in the preoperative diagnosis and management of pancreatic cysts $>3 \mathrm{~cm}$. Cancer Cytopathol 2014; 122: 804-809

7 Hong S-KS, Loren DE, Rogart JN et al. Targeted cyst wall puncture and aspiration during EUS-FNA increases the diagnostic yield of premalig- nant and malignant pancreatic cysts. Gastrointest Endosc 2012; 75: $775-782$

8 Barresi L, Tarantino I, Fabbri $C$ et al. Technique of FNA and biopsy by using a needle with side fenestration in pancreatic cystic lesions. Gastrointest Endosc 2014; 80: 897-898

9 Barresi L, Tarantino I, Traina $M$ et al. Endoscopic ultrasound-guided fine needle aspiration and biopsy using a 22-gauge needle with side fenestration in pancreatic cystic lesions. Dig Liver Dis 2014; 46: 45 - 50

10 Sendino O, Fernández-Esparrach G, Solé M et al. Endoscopic ultrasonography-guided brushing increases cellular diagnosis of pancreatic cysts: a prospective study. Dig Liver Dis 2010; 42: 877-881

11 Lozano MD, Subtil JC, Miravalles TL et al. EchoBrush may be superior to standard EUS-guided FNA in the evaluation of cystic lesions of the pancreas: preliminary experience. Cancer Cytopathol 2011; 119: 209214

12 Aparicio JR, Martínez J, Niveiro $M$ et al. Direct intracystic biopsy and pancreatic cystoscopy through a 19-gauge needle EUS (with videos). Gastrointest Endosc 2010; 72: 1285-1288 